\title{
ICWMMN2006 Proceedings
}

\section{A NEW FORMULA for the ANGLE-of-ARRIVAL DISTRIBUTION in MOBILE ENVIRONMENT}

\author{
Khoa N. Le \\ Griffith University, Griffith School of Engineering, Gold Coast, Australia \\ Email: K.Le@griffith.edu.au
}

\begin{abstract}
This paper theoretically derives an angle-of-arrival (AoA) distribution in multi-path mobile environment where the scatterer distribution obeys a hyperbolic sech function. Detailed comparisons with the well-known Gaussian distribution are given using simulations for varying distance between the base station (BS) and user equipment (UE) and the UE's effective radius.
\end{abstract}

Keywords: antenna, hyperbolic distribution, Gaussian distribution, angle-of-arrival.

\section{Problem definition}

Consider an antenna system shown in Figure 1 from which a base station (BS) is located a distance $D$ from the user equipment's (UE) antenna [1]. The effective radius of the UE is $R_{\text {eff }}$ which encloses a circular area within which random scatterers obey the hyperbolic distribution as shown. Evidently, the AoA at the BS is dependent on $D$ and $R_{\text {eff. }}$.

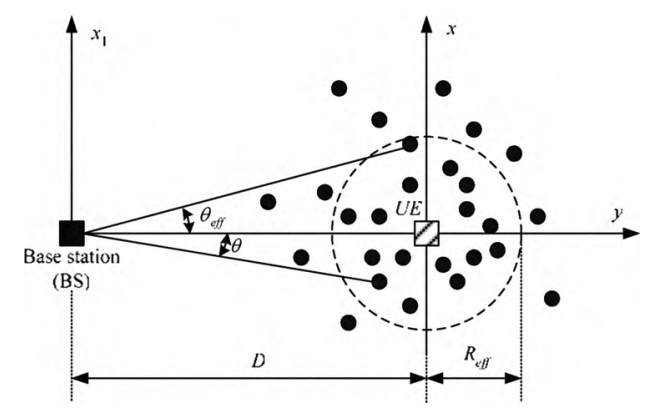

Figure 1: A schematic diagram of $\theta$ with random scatterers.

The AoA $\theta$ seen at the BS is assumed to be small so that

$$
\sin (\theta) \approx \theta \text {. }
$$

\section{Hyperbolic and Gaussian distributions}

The hyperbolic and Gaussian distributions are mathematically given as and Error! Reference source not found.) and Error! Reference source not found.) respectively [2-7]

$$
p_{G}(x)=\frac{\exp \left(-\frac{(x-\mu)^{2}}{2 \sigma^{2}}\right)}{\sigma \sqrt{2 \pi}},
$$

with variance of $\sigma$ and mean $\mu$,

$$
p_{H}(x)=\frac{\beta-\mu}{\pi} \operatorname{sech}[(\beta-\mu) x] \text {, }
$$

with variance of about $2.4674 / \beta^{2}$ and mean of $\mu$. It should be stressed that both distributions resemble the bell-shaped curve which is ideal for angle-of-arrival modelling.

\section{Derivation of the AoA using the hyperbolic distribution to model random scatterers}

Mathematically, the probability density function (pdf) of the AoA is expressed as [1]

$p(r, \varphi)=\frac{1}{\pi R_{e f f}^{2}} \operatorname{sech}\left(\frac{r^{2}}{R_{e f f}^{2}}\right)$,

where $(r, \varphi)$ are the polar coordinates centred at the UE, $r$ is the distance to a given scatterer from the UE, and $R_{\text {eff }}$ is the distance at which the pdf decreases by a factor of $e$,

By converting the pdf given in Eq. (3) to the polar coordinates $(R, \theta)$ located at BS resulting in $x_{1}=x=R \sin \theta$ and $y_{1}=R \cos \theta$. Using the Jacobian transformation, one obtains [1]

$$
\begin{aligned}
& p(R, \theta)=\frac{R}{\pi R_{e f f}^{2}} \\
& \operatorname{sech}\left(\frac{R^{2}-2 R D \cos (\theta)+D^{2}}{R_{\text {eff }}^{2}}\right),
\end{aligned}
$$

where $R$ is the new variable in the polar coordinates at BS.

To obtain the pdf of $\theta$, one has to eliminate $R$, which means

$$
p(\theta)=\int_{0}^{+\infty} \frac{R}{\pi R_{e f f}^{2}}
$$

$\operatorname{sech}\left(\frac{R^{2}-2 R D \cos (\theta)+D^{2}}{R_{e f f}^{2}}\right) d R$

After lengthy mathematical manipulation and simplifications, the theoretical expression of the AoA using hyperbolic random scatterers is then given by 


$$
\begin{gathered}
I=\frac{1}{\pi} \sum_{m=1}^{+\infty}\left[\begin{array}{l}
(-1)^{\frac{m-1}{2}} \exp \left(-m \tau^{2}\right) \\
\tau \sqrt{\frac{\pi}{m}} \cos (\theta) \exp \left[m \tau^{2} \cos ^{2}(\theta)\right] \\
\{1+\operatorname{erf}[\tau \sqrt{m} \cos (\theta)]\}
\end{array}\right] \\
=\frac{1}{\pi} \sum_{m=1}^{+\infty}(-1)^{\frac{m-1}{2}} \exp \left(-m \tau^{2}\right) \frac{1}{m} \\
+\frac{\tau \cos (\theta)}{\sqrt{\pi}} \sum_{m=1}^{+\infty}(-1)^{\frac{m-1}{2}}\left(\frac{1}{\sqrt{m}}\right) \\
\exp \left[-m \tau^{2} \sin ^{2}(\theta)\right]
\end{gathered}
$$

which is plotted in Figure 2 as a function of $\theta$ and $\tau=$ $D / R_{\text {eff }}$, resembling the famous bell-shaped curve reported in [1]. This means that the hyperbolic distribution can be effectively employed to study the AoA in mobile environment which has recently become a popular research topic.

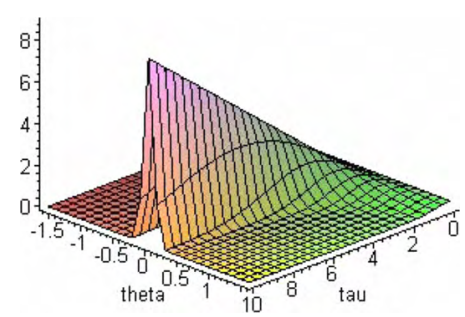

Figure 2: Theoretical hyperbolic AoA distribution as a function of $\tau=D / R_{\text {eff }}$ and $\theta$

\section{Performance comparison of the hyperbolic and Gaussian distributions}

To thoroughly study the AoA distribution using the Gaussian and hyperbolic distributions, computer simulations are used when $R_{\text {eff }}$ and $D$ are varied. From that, different AoA distributions under different conditions and performance comparisons can be clearly revealed.

\subsection{Performance comparisons when selecting $\boldsymbol{R}_{\text {eff }}$}

Figure 3 to Figure 6 graphically express the AoA as a function of $\tau$ and $\theta$ as the radius $R$ varies. From Figure 3, for $R_{e f f}=1$, it can be seen that the hyperbolic AoA reveals more information on the AoA distribution, however, it suffers from discontinuity for large $D$, which is the distance between the user equipment (UE) and the base station (BS). By comparison, as can be seen from Figure 4, the Gaussian distribution yields a smooth and continuous AoA distribution for $0 \leq D \leq 20$ compared to the coverage distance of $0 \leq D \leq$ 60 by using the hyperbolic distribution. Figure 5 and Figure 6 show the hyperbolic and Gaussian AoA distributions for $R_{\text {eff }}=$ 30 which can be considered as the maximum radius that a random scatterer can be away from the UE. It is clear that from Figure 3 to Figure 6, the performance of the hyperbolic distribution decreases as $R_{\text {eff }}$ increases, but that of the Gaussian distribution increases. In addition, thus, there is a strong trade-off between the coverage distance $D$ and the quality of coverage signals, i.e. the larger the coverage distance $D$, the lower the coverage quality by using the hyperbolic distribution to model random scatterers. The coverage quality is represented by the discontinuity of hyperbolic AoA distribution over large values of $D$. Currently, more work is under progress to quantitatively estimate the performance of the hyperbolic and Gaussian distributions as $R_{\text {eff }}$ and $D$ vary.

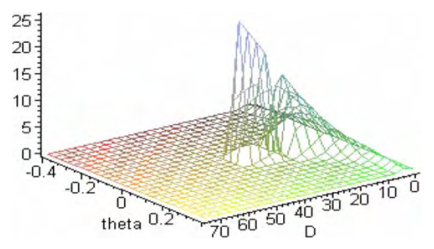

Figure 3: Hyperbolic AoA distribution with $R_{\text {eff }}=1$, the discontinuous operational range is $20 \leq D \leq 30$

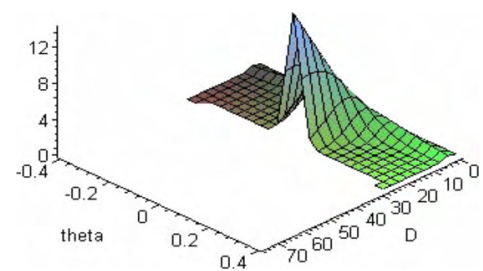

Figure 4: Gaussian AoA distribution with $R_{\text {eff }}=1$, the operational range is $0 \leq D \leq 25$

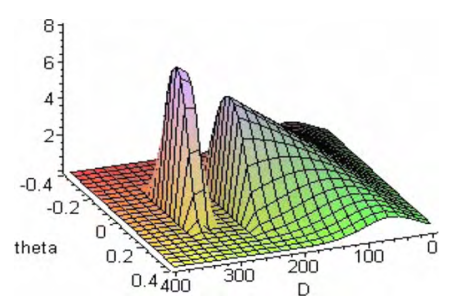


Figure 5: Hyperbolic AoA distribution with $R_{e f f}=30$, the discontinuous operational range is in $200 \leq D \leq 250$

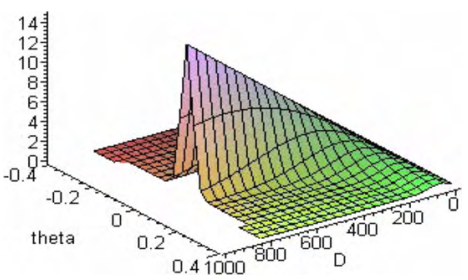

Figure 6: Gaussian AoA distribution with $R_{\text {eff }}=30$,

the operational range is in $0 \leq D \leq 800$

To further examine the behaviour of the AoA distribution, a systematic approach is employed by locating "poles" and "zeros" of a transfer function of a system in which $D$ is assumed to be the "output" and $R_{\text {eff }}$ the "input". Figure 7 plots the coverage distance $D$ on a log-log graph as $R_{\text {eff }}$ varies from 1 to 10 . At $R_{\text {eff }} \approx 3.3$, the two distributions have an approximate identical coverage distance $D$. It should be noted that Figure 7 provides insights into the behaviour of the $D$ in terms of $R_{e f f}$. From that, it is possible to work out the optimum value of $D$ under some given $R_{\text {eff }}$ and vice versa. More work is currently in progress as to model $D$ as a function of $R_{\text {eff }}$ using Pade approximation.

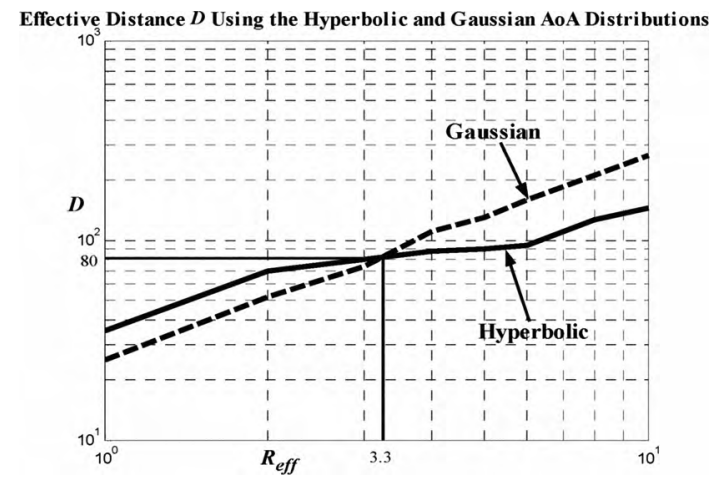

Figure 7: Coverage distance $D$ between BS and UE's antenna using the hyperbolic and Gaussian AoA distributions when 1

$$
\leq R_{e f f} \leq 10
$$

\subsection{Performance comparisons when selecting D}

By varying $D$, the received signal at BS from the UE's antenna is varied because of the distance and the number of scatterers. Thus, it is important to measure the simulated received signal power at $\mathrm{BS}$, which determines the effectiveness of the distributions. Figure 8 and Figure 9 plot the Gaussian and hyperbolic AoA distributions as a function of $R_{\text {eff }}$ and $\theta$ with $D=1$, from which it is clear that the received signal power at BS using the Gaussian distribution is smaller than using the hyperbolic distribution. Figure 10 and Figure 11 plot the Gaussian and hyperbolic AoA distributions for $D=50$, from which it is clear that both distributions suffer from non-operational mode for small $R_{\text {eff }}$ and large $D$. For $R_{\text {eff }}$ $\leq 2$, the hyperbolic distribution yields about $4 \mathrm{~dB}$ stronger in received signal power, and for $43 \geq R_{\text {eff }}>2$, it yields on average $1 \mathrm{~dB}$ stronger in received signal power than the Gaussian distribution. It should also be noted that for a small coverage distance $D$, the AoA distribution is less stable than when $D$ is large as visible oscillations are observed as seen in Figure 8 and Figure 9 for $D=1$. Figure 12 plots the received signal power at the BS for both distributions from which it is clear that the hyperbolic distribution outperforms the Gaussian distribution for $D \leq 43$ approximately. For $D>43$, the hyperbolic distribution suffers from a sudden drop of power while the received power using the Gaussian distribution remains strong.

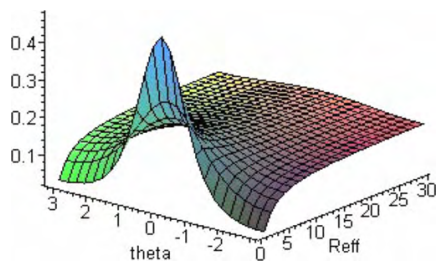

Figure 8: Gaussian AoA distribution as a function of $R_{\text {eff }}$ and $\theta$ with $D=1$

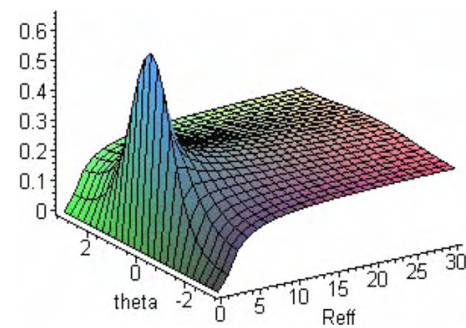

Figure 9: Hyperbolic AoA distribution as a function of $R_{\text {eff }}$ and $\theta$ with $D=1$

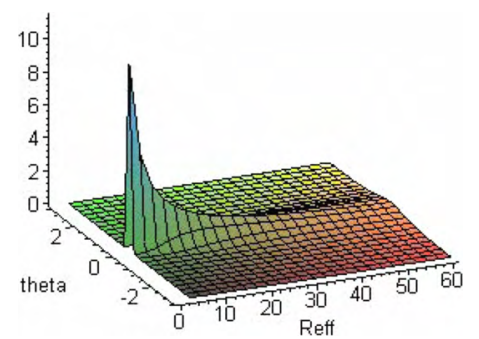

Figure 10: The Gaussian distribution with $D=50$ 


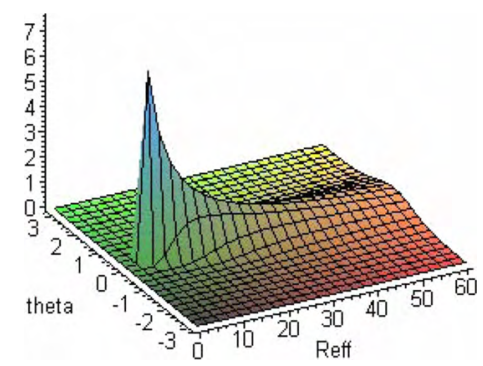

Figure 11: The hyperbolic distribution with $D=50$

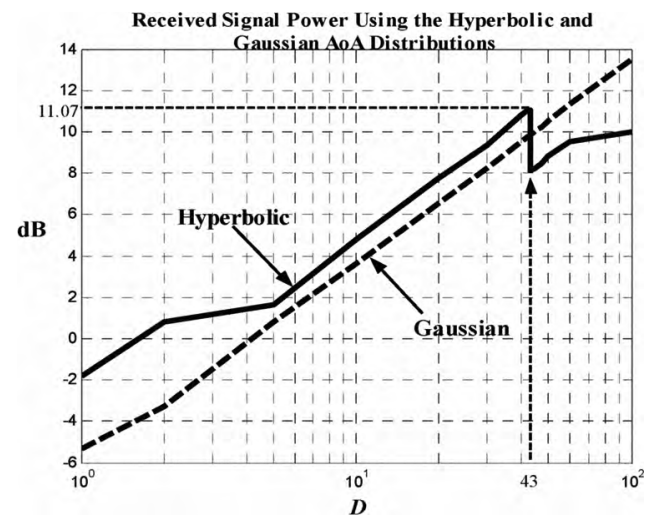

Figure 12: Received signal power in $\mathrm{dB}$ using the hyperbolic and Gaussian AoA distribution when $D$ is varied. Pade approximation method can be used to gain further insight into the behaviour of the received signal power and $D$.

\section{Conclusion}

The angle-of-arrival (AoA) in multi-path mobile environment has been derived using a new hyperbolic distribution as a scatterer distribution. This paper has demonstrated that the AoA distribution derived from the hyperbolic distribution for random scatterers is more effective than the AoA distribution derived from the Gaussian distribution. It has been shown that the hyperbolic AoA distribution is more effective than the Gaussian AoA distribution when $R_{\text {eff }} \leq 3.3$. For $R_{\text {eff }}>3.3$, the latter outperforms the former by providing a longer coverage distance $D$. For $3.3<R_{\text {eff }} \leq 28$, the hyperbolic AoA distribution can be equivalently considered as the Gaussian AoA distribution and thus it can be used to study the AoA in mobile environment. It has also been shown that the hyperbolic AoA distribution yields larger received signal power than the Gaussian distribution for $D \leq 43$. Another point this paper is trying to show is that there exist more effective distribution(s) to model random scatterers than the Gaussian distribution which has been widely used in the literature.

Log-log plots on the relationships between $R_{\text {eff }}$ and $D$, and $D$ and received signal power yield further insight into these parameters by using a Pade approximation method to derive their polynomial expressions. From that, "poles" and "zeros" of a transfer function can be worked out and studied. More work is currently in progress to further explore in-depth relations among these parameters. Further work on how to measure the amount of information yielded by the hyperbolic and Gaussian AoA distributions is currently in progress. Additional work on the effects of isotropic in the Gaussian distribution and semi-isotropic in the hyperbolic distribution is currently being carried out and will be reported in a separate publication.

\section{References}

1. Bevan, D.D.N., et al., "Gaussian Channel Model for Mobile Multipath Environment," EURASIP Journal on Applied Signal Processing, 2004, 2004(9), pp. 1321-9.

2. Choi, H.I. and Williams, W.J., "Improved timefrequency representation of multi-component signals using exponential kernels," IEEE Transactions on Signal Processing, 1989, 37(6), pp. 862-71.

3. Le, K.N., "Time-frequency distributions for crack detection in rotors -- A fundamental note," Journal of Sound and Vibration, 2006, 294(1-2), pp. 397-409.

4. Le, K.N., Dabke, K.P., and Egan, G.K., "Hyperbolic kernel for time-frequency power spectrum," Optical Engineering, 2003, 42(8), pp. 2400-15.

5. Le, K.N., Dabke, K.P., and Egan, G.K., "On mathematical derivation of auto-term functions and signal-tonoise ratios of Choi-Williams, first- and nth-order hyperbolic kernels," Digital Signal Processing: A Review Journal, 2006, 16(1), pp. 84-104.

6. Le, K.N., Dabke, K.P., and Egan, G.K., "Signal detection using non-unity kernel time-frequency distributions," Optical Engineering, Dec. 2001, 40(12), pp. 2866-77.

7. Perdersen, K.I., Mogensen, P.E., and H., F.B., "A Stochastic Model of the Temporal and Azimulthal Dispersion Seen at the Base Station in Outdoor Propagation Environments," IEEE Transactions on Vehicular Technology, 2000, 49(2), pp. 437-47. 